\title{
XXII. On the determination in absolute units of the intensities of powerful magnetic fields
}

\author{
A. Gray M.A. F.R.S.E.
}

To cite this article: A. Gray M.A. F.R.S.E. (1883) XXII. On the determination in absolute units of the intensities of powerful magnetic fields, Philosophical Magazine Series 5, 16:98, 144-156, DOI: $10.1080 / 14786448308627410$

To link to this article: http://dx.doi.org/10.1080/14786448308627410

曲 Published online: 28 Apr 2009.

Submit your article to this journal $[\pi$

Џll Article views: 2

Q View related articles ๘ 
I may refer to my paper in the Philosophical Magazine for September 1871 for some investigations which confirm Mr. Ferrel's results.

Bushmills, co. Antrim, July 20, 1883.

XXII. On the Determination in Absolute Units of the Intensities of Powerful Magnetic Fields. By A. Grax, M.A., F.R.S.E., Chief Assistant to the Professor of Natural Philosophy in the University of Glasgow*.

SO far as I know, no method has yet been published by $D$ which the intensity of a powerful magnetic field, such as that in the space in which the armature-coils of a dynamo move between the poles of the field-magnets, can be determined in absolute measure; and inventors of dynamos and other experimenters in practical electricity have hitherto, at a great expense of time and money, had to construct their actual apparatus, and find by the results obtained in actual practice the efficiency or non-efficiency of their arrangements. So much so has this been the case that it has even been held that the absolute value of the intensity of a magnetic field is a thing with which practical electricians have no concern, that, although theoretically it may have some importance, it has no application in practice. That all measurements, whether of electric or of magnetic quantities, should be expressed in absolute units-that is, in units altogether independent of the locality, surroundings, and apparatus of the experimenter-is, I think, of very great importance, if these results are to be of any service to others. And it would; I feel sure, be a great benefit to all engaged in practical electrical work, if the intensities of the magnetic fields obtainable with various forms of electromagnets, made with different kinds of iron, were determined in absolute units and published with full particulars of the apparatus. But failing the knowledge which would be derived from experiments such as these, a very great saving of time and money might be made if the inventor of a dynamo, for example, were to first make models of his magnets on a small scale, and determine the magnetic-field intensities obtainable in them with different current-strengths, and then to reason from the results in the model to those in the full-sized machine. The object of the present paper is to describe some methods by which the intensity of a powerful magnetic field may be determined in absolute units. The methods are wholly due to Sir William Thomson ; but for the sketch of theory given

* Communicated by the Author. 
below for each, the details of the experimental arrangements, and the results $\mathrm{I}$ am alone responsible.

(a) The first method, which is somewhat interesting theoretically, is one which was used in the determination of the magnetic field-intensities in the space in which the signal-coil is suspended in some of Sir William Thomson's Siphon Recorders made with permanent magnets. In it advantage is taken of the signal-coil, which consists of a rectangular coil a little more than 5 centim. long and 2 centim. broad, made of thin wire and supported by a silk thread above, so as to hang in a vertical plane round a rectangular core of iron, which nearly fills, but nowhere touches, the coil. To the lower end of the coil two silk threads are attached, as shown in the diagram, and are stretched against a bridge $B$ by two weights resting on the inclined plane $W$. This bifilar arrangement gives a directive force, tending to bring the plane of the coil into parallelism with that of the bifilar threads; so that when the coil is disturbed from that position, which is one of stable equilibrium, and then left to itself, it will, if the circuit be not closed, vibrate about the position of equilibrium with a determinate period of oscillation, with slowly diminishing range, until at last it comes to rest.

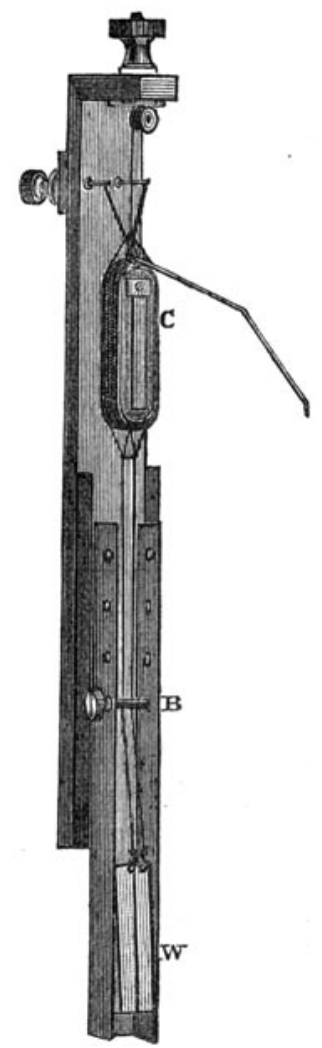
But if the circuit be closed through a high resistance, the coil will come more rapidly to rest; and if we gradually diminish this resistance, deflecting the coil through the same angle and noting its subsidence at each diminution, we shall find it come more and more quickly to rest, until a resistance is obtained with which in circuit it just returns to the position of equilibrium without passing that position. When this resistance has been determined, the strength of the field can be calculated.

Let $\theta$ be the deflection of the coil from the position of equilibrium at time $t$, and $\mathrm{T}$ its period of oscillation when the circuit is not closed. We have then, neglecting the resistance of the air and other disturbances, for the equation of motion,

$$
\frac{d^{2} \theta}{d t^{2}}+\frac{4 \pi^{2}}{T^{2}} \theta=0 . \quad \text {. . . . . . }
$$


Let now the circuit of the coil be closed; a retarding force due to the current induced in the wire, and, if the effect of self-induction be neglected, proportional to the angular velocity, will act on the coil ; and the equation of motion for this case will be of the form

$$
\frac{d^{2} \theta}{d t^{2}}+k \frac{d \theta}{d t}+\frac{4 \pi^{2}}{\mathrm{~T}^{2}} \theta=0 . \quad \text {. . . . . . }
$$

For let $I$ be the mean intensity of the magnetic field over the space occupied by the coil at time $t, \mathrm{M}$ the electromagnetic inertia (coefficient of self-induction) of the circuit for that position of the coil, $R$ the total resistance in the circuit, $\mu$ the moment of inertia of the coil round a vertical axis passing through its centre, $L$ the effective length of wire in the coil (that is, the length of wire in its two vertical sides), and $b$ the mean half-breadth of the coil. If we call $\mathrm{N}$ the number of lines of force which pass through the coil at time $t$, and $\gamma$ the strength of the induced current in the coil at that instant, we have plainly

$$
\mathrm{N}=b \mathrm{IL} \sin \theta-\mathrm{M} \gamma
$$

The rate at which $\mathrm{N}$ increases per unit of time is therefore

$$
\frac{d \mathrm{~N}}{d t}=b \mathrm{IL} \cos \theta \frac{d \theta}{d t}+b \mathrm{~L} \sin \theta \frac{d \mathrm{I}}{d t}-\frac{d}{d t}\left(\mathrm{M}_{\gamma}\right) ;
$$

and if $\theta$ be small, and I be therefore supposed the same for every position of the coil, we have approximately

$$
\frac{d \mathrm{~N}}{d t}=b \mathrm{IL} \frac{d \theta}{d t}-\frac{d}{d t}\left(\mathrm{M}_{\boldsymbol{\gamma}}\right) \text {. }
$$

But $\frac{d \mathrm{~N}}{d t}$ is the electromotive force due to the inductive action; hence the current $\gamma$ is by Ohm's law given by the equation

$$
\gamma=\frac{b \mathrm{IL}}{\mathrm{R}} \frac{d \theta}{d t}-\frac{1}{\mathrm{R}} \frac{d}{d t}\left(\mathrm{M}_{\gamma}\right)
$$

It was assumed that the second term of this expression for $\gamma$ would prove negligible in comparison with the first; and this assumption was so far justified by the results of the experiments, which agreed fairly well with results obtained, for other instruments of the same pattern, by a modification of the second method described below.

The couple due to the action of the field on the current is $b I \mathrm{~L} \gamma$; and therefore, on the supposition of negligible selfinduction, the retardation of the angular velocity of the coil at time $t$ is

$$
k \frac{d \theta}{d t}=\frac{b^{2} \mathrm{I}^{2} \mathrm{~L}^{2}}{\mu \mathrm{R}} \frac{d \theta}{d t}
$$


Hence (2) becomes

$$
\frac{d^{2} \theta}{d t^{2}}+\frac{b^{2} \mathrm{I}^{2} \mathrm{~L}^{2}}{\mu \mathrm{R}}-\frac{d \theta}{d t}+\frac{4 \pi^{2}}{\mathrm{I}^{2}} \theta=0 . \quad . \quad . \quad .
$$

The motion represented by this differential equation will be oscillatory or non-oscillatory, according as the roots of the auxiliary quadratic are imaginary or real-that is, according as $\frac{4 \pi}{\mathrm{T}}>$ or $<\frac{b^{2} \mathrm{I}^{2} \mathrm{~L}^{2}}{\mu \mathrm{R}}$. Hence, if $\mathrm{R}$ be the critical resistance at which the motion just ceases to be oscillatory, we have

$$
\mathrm{I}^{2}=\frac{4 \pi \mathrm{R} \mu}{\mathrm{T} b^{2} \mathrm{~L}^{2}} . \quad . \quad . \quad . \quad .
$$

When $\mathrm{L}$ and $b$ are expressed in centimetres, $\mu$ in grammes and centimetres, $T$ in seconds, and $R$ in centims. per second, $\mathrm{I}$ is given by this equation in absolute C.G.S. units of magnetic field-intensity.

The method of experimenting consisted in first finding the value of $\mathrm{T}$, the free period of vibration of the coil with its circuit uncompleted, then finding the resistance which, being placed in circuit with the coil, just brought the needle to rest without oscillation. This resistance was conveniently obtained by means of a resistance-box included in the circuit, and therefore added no self-induction to that in the coil. An aluminium arm attached to the coil, and carrying the siphon, served as an index to render the motions of the coil visible. The resistance $\mathbf{R}$ was first made much too great, so as to give a slow subsidence, then gradually diminished until the value which just prevented oscillation was reached; and it was found that this value could be determined easily within 50 ohms, and, with great care, to $20 \mathrm{ohms}$. As the experiments on the recorders had to be made somewhat hurriedly, and, on account of the disturbances, neglected, and, further, as $\mu$ was taken as equal to $W b^{2}$, where $W$ is the mass of the coil, the results could not be taken as giving more than a rough approximation to $I$; but those for two instruments are given below in illustration of the method. For both instruments the values of $\mathrm{W}, \mathrm{L}$, and $b$ were the same, and were respectively taken as $3 \cdot 343$ grammes, 3338 centim., and $\cdot 95$ centim. Each coil had a mean vertical length of 5.3 centim., a mean breadth of 1.9 centim., and contained 45.72 metres of fine wire arranged in 290 turns, and had a resistance of about $500 \mathrm{ohms}$.

$\mathrm{T}$.

(1) $\cdot 465 \mathrm{sec}$.

(2) .500 ,

$\mathrm{R}$

$3330 \times 10^{9}$ centim. per sec. $3530 \times 10^{9} \quad, "$,

$(\beta)$ The second method is one which was adopted in measurements which I made of the fields of some experimental elec- 
tromagnets, and it depends on the action between a magnetic field and a conductor in the field carrying a constant current. The ends of the cores of the electromagnets were long straight surfaces of iron, separated from the surface of iron placed opposite to them by only a narrow space at each pole. In the experiments, of which I give some results below, the intensity of the field was found, in a space about 2 millim. wide, between one end of the iron core of an electromagnet and the surface of a thick piece of iron. The length of the pole-face was 30 centim. The magnet and corresponding iron were placed with their faces vertical ; and a copper wire was suspended vertically in the space between them, and insulated from their surfaces. The lower end of the wire dipped into a mercury-cup, and its upper end into a second mercurycup on the top of the electromagnet, without touching the sides or bottoms of the cups. By means of these cups the wire could either be put in series with the coil of the electromagnet or in circuit with an independent battery or generator. The wire was hung in a vertical position (as shown in the diagram).

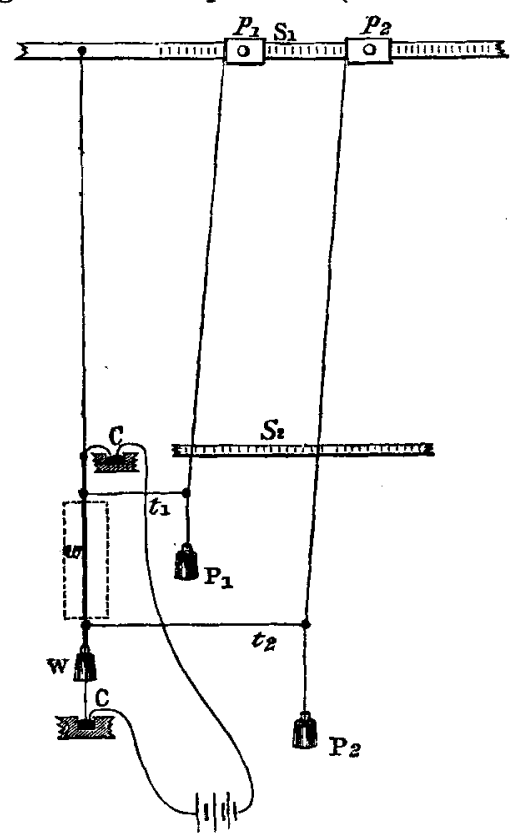

from the lower end of a cord about two metres long, attached to a firm support above, and was kept taut by two half-pound weights (represented by the single weight $W$ in the diagram) hung at the ends of a cross bar firmly attached to the wire. The position of the wire was adjusted by shifting the point of 
attachment of the cord above until the wire was exactly opposite two marks, one at the upper end and one at the lower end of the vertical central line of the pole-face. A thin thread, $t_{1}$, was attached to the wire just above the electromagnet, and carried out to one side nearly horizontally, and attached by its other end at a point near the lower end of the cord of the pendulum, $\mathrm{P}_{1}$. A second thread, $t_{2}$, was attached to the lower end of the wire, and carried out parallel to the thread $t_{1}$, and attached to a second pendulum, $\mathrm{P}_{2}$, at a somewhat greater distance from the wire. The pendulums, $\mathrm{P}_{1}, \mathrm{P}_{2}$, were simply weights, in some cases of half a pound each, and in other cases 100 grammes each, hung by fine cords from one to two metres long from blocks, $p_{1}, p_{2}$, which could be moved along a horizontal scale, $\mathrm{S}_{1}$, placed nearly in a vertical plane passing through the wire at right angles to the coil of the electromagnet, and therefore parallel to the pole-face. A second scale, $S_{2}$, placed just above the point of attachment of the upper thread, and nearly in the same vertical plane as $S_{1}$, gave the position at any time of a point in each of the pendulum-cords at the same distance below the point of suspension.

The method of performing an experiment was as follows:The vertical positions of the cords of the pendulums when the threads $t_{1}, t_{2}$ were quite slack and the wire was in position were read off from the seales. A current was then sent through the wire and coil in series. The electromagnetic action between the current in the wire and the magnetic field caused the wire to move bodily to one side across the lines of force; and the direction of the current in the wire was so arranged that the side towards which the wire moved was that remote from the pendulums. By moving out the blocks, $p_{1}, p_{2}$, pull (in consequence of the deviation of the pendulums from the vertical) was brought to bear through the threads $t_{1}, t_{2}$ on the upper and lower ends of the wire; and this was gradually increased until the wire was brought back as nearly as possible to its former vertical position. The readings given by the pendulums on the two scales were then taken, and at the same time a reading of the current-strength on the current-galvanometer. The difference between the two motions on $S_{1}$ and $S_{2}$ for either pendulum, divided by the distance between the scales, gave the tangent of the inclination of the pendulum to the vertical.

Let, now, I be the mean intensity for the position of the wire of the magnetic field in C.G.S. units, $\gamma$ the current flowing on the wire also in C.G.S., L the length of the wire in the field in centims., $F$ the force per unit of length on the wire in dynes, we have

$$
\mathrm{I} \gamma=\mathrm{F} \text {. }
$$


But the total horizontal force exerted by the pendulums is $\mathrm{W}_{1} g \tan \theta_{1}+\mathrm{W}_{2} g \tan \theta_{2}$, where $\mathrm{W}_{1}, \mathrm{~W}_{2}$ are taken in grammes, and $g$, the acceleration produced by gravity, as approximately 981 centim. per second per second. Hence we have

$$
\mathrm{I}=\frac{g}{\gamma \mathrm{L}}\left(\mathrm{W}_{1} \tan \theta_{1}+\mathrm{W}_{2} \tan \theta_{2}\right) . . .
$$

From this formula the values of I can be calculated from the values of the other quantities found by experiment. The following are the results of some actual experiments made in September last:-

\begin{tabular}{|c|c|c|c|c|c|}
\hline & $\begin{array}{c}\mathrm{W}_{1} \text {, } \\
\text { in grammes. }\end{array}$ & $\begin{array}{c}\mathrm{W}_{2} \text {, } \\
\text { in grammes. }\end{array}$ & in C.G.S. $^{\prime}$ & $\begin{array}{c}\mathrm{I} \\
\text { in C.G.S. }\end{array}$ & $I / \gamma^{*}$ \\
\hline (1) ... & 100 & 100 & .09 & 2210 & 24555 \\
\hline (2). & " & " & $\cdot 132$ & 3410 & 26590 \\
\hline (3) & $"$ & , & $\cdot 140$ & 4170 & $29785(?)$ \\
\hline (4) & $"$ & , & $\cdot 156$ & 4373 & 28032 \\
\hline (5) $\ldots \ldots \ldots$ & $"$ & $"$ & $\cdot 172$ & 4864 & 28283 \\
\hline
\end{tabular}

The same method was applied to find the value of I for different parts of the field on the two sides of the middle position. With two operators (one to move the pendulums, the other to watch the wire and take the galvanometerreadings) the experiments were quickly and satisfactorily performed.

The following modification of the same method has been used for the determination of $I$ for several recorder magnets, with results agreeing well with those obtained (for other instruments) by the first method described above. A horizontal bar, carrying a divided scale, was fixed parallel to the equilibrium position of the plane of the coil, in a vertical plane passing through the glass siphon a little beyond the point of the aluminium arm supporting it. A single block, to which the upper end of a pendulum-thread was attached, was made movable along the horizontal bar, so that any displacement of the point of suspension could be observed by means of the divided scale. The pendulum was made of a brass weight, generally of one gramme, as bob, hung by a very fine silk thread long enough to allow the bob to hang a few centimetres below the siphon. When the coil was in the position of equilibrium, the pendulum was moved until the thread just rested against the siphon without deflecting it, and the reading on the scale was noted. A current from one or two Daniell's cells was then sent through the coil so as to deflect it from the equilibrium

* This column is given, as $\gamma$ was also the magnetizing current. 
position in the direction to cause the siphon to press against the thread. The point of suspension of the pendulum was then moved so as to restore the coil to its initial position, find the distance through which it was moved noted. This distance, divided by the vertical height of the point of suspension above the point at which the thread touched the siplon, gave the tangent of the inclination of the thread to the vertical; and this multiplied by the downward pull in dynes on the weight gave the horizontal force restoring the coil to its equilibrium position.

When equilibrium had been obtained, the difference of potentials between the terminals of the coil was immediately measured in volts by means of a potential-galvanometer previously arranged in readiness; and from the result and the known resistance of the coil the current flowing was deduced in C.G.S. units. The mean intensity $I$ of the field over the space occupied by the coil in the equilibrium position was tisen easily calculated. For let $\gamma$ be the current in the coil, $\theta$ the inclination of the pendulum-thread above the siphon to the vertical, $l$ the distance in centimetres of the point at which the thread touched the siphon from the vertical axis round which the coil turned, and $W$ the pendulum-weight in grammes. Using $\mathrm{L}$ and $b$ with the same meaning as before, we have plainly the equation of equilibrium

and therefore

$$
\begin{aligned}
\mathrm{IL} b \gamma & =\mathrm{W} g l \tan \theta, \\
\mathrm{I} & =\frac{\mathrm{W} g l}{\mathrm{~L} b \gamma} \tan \theta . \quad . \quad . \quad . \quad .
\end{aligned}
$$

The method of determining a magnetic field-intensity by measuring the electromagnetic action on a conductor carrying a current in the field, may be very conveniently applied to the space between the opposite poles of a field magnet in which the armature-coils of a dynamo are made to revolve. A rectangular frame of stout copper wire or narrow copper strip, containing one or more turns (insulated from one another if there are more than one), is constructed of such a size that two opposite sides are at a distance apart equal to the diameter of the armature, and of length equal to the length of the pole-faces. The rectangle, hould begin and end near the middle of the two other sides; and the two ends of the wire should be brought out side by side, insulated from one another, at right angles to the side, a distance of two or three inches. A pair of knife-edges, which may be made for ease of a piece of hardened copper or brass wire filed so as to have a nearly triangular section, are soldered, one at the centre of the side where the ends of the wire are brought out, the other at the centre of the opposite side; so that the rectangle is symme- 
trical on the two sides of the knife-edges, and is horizontal when the knife-edges are turned downwards. The sides on which the knife-edges are placed may conveniently be slightly bent so as to have an upward convexity, in order that the line of knife-edges mav, when the wire frame is made to rest on them, be a little above the centre of inertia of the system, in order that the frame may rest stably in the horizontal position; or the frame may be loaded so as to have sufficient stability by pieces attached below the knife-edges. The ends of the wire are to be bent first a little up, then downwards so that the point of one is about an inch nearer to the frame than the other, and both points are nearly in the line through the knife-edges.

The field-magnets are set up so that the plane cutting their pole-faces in which the field-intensity is to be measured is horizontal ; and two supports, carrying metal V-shaped rests for the knife-edges, are arranged so that the frame when resting on its knife-edges has the two sides lying along the polefaces in the horizontal plane through the two parts of these faces near which the field is to be determined. Two cups containing mercury are arranged on a support so placed that the ends of the wire of the frame dip into the mercury, without touching the sides or bottoms of the cups. One terminal of a convenient battery is connected to one of the cups; and the other terminal is so arranged that the circuit can be completed by depressing the terminal into the other cup, or broken by raising it, when required.

When an experiment is made, the magnets are excited by a current of the required amount, and a measured current is sent round the frame, which is made to turn on its knife-edges by the electromagnetic action. A weight which can be slided along the frame is hung on one of the sides which carry the knife-edges, and moved to such a distance from the line of knife-edges that the frame is brought back to its initial position. This distance in centimetres and the mass of the weight in grammes are carefully determined, with the strength of the current flowing in the frame; and the mean intensity of the field over the space occupied by the part of the frame in the field calculated from the results. Let I be this mean intensity, $\mathrm{I}$ the length of wire of the frame in the field, $b$ the horizontal distance in centimetres of each side from the line of knifeedges, $\mathrm{W}$ the mass of the equilibrating weight in grammes, and $r$ its distance from the line of knife-edges, $g$ the acceleration produced by gravity (=981 centim. per second per second), and $\gamma$ the strength of the current in C.G.S. units ; we have by equating moments,

$$
\mathrm{I}=\frac{\mathrm{W} r}{\mathrm{~L} \gamma} g . \quad . \quad . \quad . \quad . \quad . \quad .
$$


If instead of a single weight, $\mathrm{W}$, several weights, $\mathrm{W}_{1}, \mathrm{~W}_{2}$, $\& c$. at distances $r_{1}, r_{2}$, \&c. be used to restore equilibrium, we have of course, instead of $\mathrm{Wr}$ in this formula, simply the sum $W_{1} r_{1}+W_{2} r_{2}+\& c$.

Another modification of this method may be convenient in some cases. The field-magnets may be so placed that the plane cutting the poles in which the intensity is to be determined is vertical, and a rectangle made of the proper dimensions and hung by a torsion-thread or wire, or by means of a bifilar, so that it is in equilibrium in the required position, and turns when deflected round a vertical axis bisecting it. The ends of the wire forming the rectangle are carried down, insulated from one another, from the middle of the lower side, and bent so that their points are in the vertical line bisecting the rectangle, and dip, like the terminals of an Ampere frame, into two mercury-cups placed one above the other on supports arranged below. Two pendulums are arranged so that their points of suspension can be moved along horizontal bars at right angles to the equilibrium position of the frame, one in the vertical plane through each side of the rectangle. These pendulums carry at the ends of light but strong threads equal known weights ; and each pulls horizontally on the corresponding side of the rectangle by means of a thread attached at one end to the middle point of the side, at the other to the pendulum-cord. The pendulums and threads are so arranged that the pulls are applied in opposite directions; and thus when the pulls are equal they give a couple tending to turn the frame round a vertical axis. Corresponding to each pendulum are a pair of horizontal scales, arranged like those shown in the second diagram above, by means of which, in the manner already described, the tangent of the inclination of the pendulum to the vertical is at once read off.

The method of experimenting is similar to that last described. The magnets are excited, and a measured current sent round the suspended rectangle, from a battery connected with the mercury-cups, in the direction to cause the rectangle to turn round in the direction opposite to the motion which the pendulums are arranged to produce. The points of suspension of the pendulums are then moved so as to restore the frame to the initial position, and the tangents of the inclinations of the cords obtained by means of the scales. If the field be symmetrical about the axis of suspension of the coil, the electromagnetic action of the frame will be a pure couple, and the equilibrating forces will form also a conple; but in general this will not be exactly the case. Let $W$ be the mass, in grammes, of each pendulum-weight, $\theta_{1}, \theta_{2}$ the inclinations of the pendulum-cords to the vertical, and let $\mathrm{L}, b, \gamma$ have the 
same meaning as before; we get by equating moments of forces,

$$
\mathrm{I}=\frac{\mathrm{W} g}{\mathrm{~L} \gamma}\left(\tan \theta_{1}+\tan \theta_{2}\right) . \quad \text {. . . . }
$$

$(\gamma)$ The third method is one which has been frequently used in the Physical Laboratory of the University of Glasgow, and consists in exploring the magnetic field by means of the induced current in a wire moved quickly across the lines of force over a definite area in the field. The wire is in circuit with a reflecting "ballistic" galvanometer-that is, a galvanometer the system of needles of which has so great a moment of inertia that the whole incluced current due to the motion of the wire has passed through the coil before the needle has been sensibly deflected. The deflection thus obtained is noted, and compared with the deflection obtained when, with the same circuit, a portion of the conductor is made to sweep across the lines of force over a definite area of a uniform field of known intensity, such as that of the earth or its horizontal or vertical component.

In performing the experiments, it is necessary to take precautions to prevent any action except that between the definite area of the field selected and the wire cutting its lines of force. For this purpose the conducting-wire, which is covered with insulating material, is bent so as to form three sides of a rectangle, one of which is of the length of the portion of field to be swept over. This side is placed along one side of the space over which it is about to be moved so that the connectingwires lie along the ends of the space; and the open rectangle is then moved in the direction of its two sides until the opposite side of the space is reached. The connecting-wires thus do not cut the lines of force, and the induced current is wholly due to the closed end of the rectangle.

Instead of a single wire cutting the lines of force, a coil of proper dimensions (for many purposes conveniently of rectangu'ar shape), the mean area of which is exactly known, may be suspended in the field with its plane parallel to the lines of force, and turned quickly round through a measured angle of convenient amount not exceeding $90^{\circ}$; or it may be suspended with its plane at right angles to the lines of force and turned through an angle of $180^{\circ}$. If $n$ be the number of turns, A their mean area, and $I$ the mean intensity of the field over the area swept over in each case, then, in the first case, if $\theta$ be the angle turned through, the area swept over is $n \mathrm{~A} \sin \theta$, and the number of lines cut is $n I A \sin \theta$; in the second, the area is $2 n \mathrm{~A}$, and the number of lines cut is $2 n \mathrm{IA}$.

In order that with the feeble intensity of the earth's field a sufficiently great deflection for comparison may be obtained, 
it is necessary that a relatively large area of the field should be swept over by the conductor. One convenient way is to mount on trunnions a coil of moderately fine wire of a considerable number of turns wound round a ring of large radius, like the coil of a standard tangent-galvanometer, and arranged with stops so that it can be turned quickly round a horizontal axis through an exaci half-turn, from a position in which its plane is exactly at right angles to the dip. This coil, of course, always remains in the circuit of the ballistic galvanometer. The change in the number of lines of force passing through the coil in the same direction relatively to the coil, produced by the half-turn, is plainly equal to twice as many times the area of the turn of mean area as there are turns in the coil (the effective area swept over) multiplied by the intensity of the field.

A sufficiently large area of the earth's field, for comparison, may otherwise be obtained very readily by carrying the wire along a rod of wood (say two or three metres long), and suspending this rod in a horizontal position by the continuations of the conductor at its ends from two fixed supports in a horizontal line at a distance apart equal to the length of the rod, and securing the remaining wires in circuit so that they may not cause disturbance by their accidental motion. The rod will thus be free to swing like a pendulum by the two suspending-wires. The pendulum thus made is slowly deflecied from the vertical until it rests against stops arranged to limit its motion. It is then quickly thrown to the other side against similar stops there, and caught. The straight conductor thus sweeps over an area of the vertical component of the earth's field equal to the product of the length of the rod into the horizontal distance between the two positions of the conductor at the extremities of its swing. The rod may be placed at any azimuth, as the suspending portions of the conductor in circuit, moving in vertical planes, can cut only the horizontal lines of force; and the induced currents thus produced have opposite directions and neutralize one another.

The calculation of the results is very simple. By the theory of the ballistic galvanometer (the same mutatis mutandis as that of the ballistic pendulum), if $q$ be the whole quantity of electricity which passes through the circuit, and if $\theta$ be the angle through which the needle has been deflected, or the "throw," we have

$$
q=\frac{2}{\mathrm{G}} \sqrt{\frac{\mu \mathrm{H}}{m}} \sin \frac{\theta}{2}
$$

where $\mu$ is the moment of inertia of the needle and attachments, $m$ the magnetic moment of the needle, $H$ the earth's horizontal magnetic force, and $G$ the constant of the galvanometer. If $\theta$ be small, as it generally Las been in these expe- 
riments, we have

$$
q=\frac{1}{\mathrm{G}} \sqrt{\frac{\mu \mathrm{H}}{m}} \cdot \theta
$$

and the quantities of electricity produced by sweeping over two areas, $A$ and $A^{\prime}$, are directly as the deflections.

Let $A$ be the area of the field or portion of field the mean intensity ( $I$ ) of which is being measured, $A^{\prime}$ and $I^{\prime}$ the same quantities for the known field, $q, q^{\prime}$ the quantities of electricity generated in the two cases, $\theta, \theta^{\prime}$ the corresponding deflections supposed both small ; we have

and therefore

$$
\begin{gathered}
q=\mathrm{AI}=\frac{1}{\mathrm{G}} \sqrt{\frac{\mu \mathrm{H}}{m}} \theta, \\
q^{\prime}=\mathrm{A}^{\prime} \mathrm{I}^{\prime}=\frac{1}{\mathrm{G}} \sqrt{\frac{\mu \mathrm{H}}{m}} \theta^{\prime},
\end{gathered}
$$

$$
\mathrm{I}=\frac{\mathrm{A}^{\prime} \theta}{\mathrm{A} \theta^{\prime}} \mathrm{I}^{\prime} \cdot \text {. . . . . . . }
$$

If convenient, $\theta$ and $\theta^{\prime}$ may be taken as proportional to the numbers of divisions of the scale traversed by the spot of light in the two cases.

I may remark, in conclusion, that the method described in (a) above gives, when the intensity of the field has been accurately measured by another method, theoretically a method of determining a resistance in absolute electromagnetic measure, This method is nearly the converse of that given by Weber, in which a magnet is made to oscillate within a coil of wire the circuit of which is closed. What I venture to propose is to hang a coil, the constants of which are known, in a sufficiently intense and uniform magnetic field, and find the decrement of the oscillatory motion produced by the induction: for it would scarcely be possible, I think, to observe with sufficient accuracy the point at which the coil just became "dead beat." How far this method may be practicable can hardly be affirmed without experiment; but I think the necessary calculations and corrections would be in some degree at least simpler than those required for the method given by Weber.

\section{Proceedings of Learned Societies. \\ GEOLOGICAL SOCIETY.}

[Continued from p. 7l.]

June 6, 1883.-J. W. Hulke, Esq., F.R.S., President, in the Chair. THE following communications were read :-

1. "The Estuaries of the Severn and its Tributaries, an Inquiry into the Nature and Origin of their Tidal Sediment and Alluvial Flats." By Prof. W. J. Sollas, M.A., F.R.S.E., F.G.S.

Various sources have been ascribed to the mud which is so charac- 